\title{
Absence d'effets d'un excès alimentaire en leucine sur les cinétiques postprandiales du glucagon et du cortisol plasmatiques chez l'agneau préruminant
}

Isabelle PAPET, Michèle GARDY-GODILLOT (*), M. DALLE (*), J. GRIZARD, M. ARNAL

Laboratoire d'Etude du Métabolisme Azoté et U.A. C.N.R.S. 041123

I.N.R.A., Theix, 63122 Ceyrat, France.

(*) Laboratoire de Physiologie Animale et U.A. C.N.R.S. 041123

Université Blaise Pascal, Clermont-Ferrand II,

24, avenue des Landais, 63170 Aubière, France.

Summary. Plasma concentrations of cortisol and glucagon have been measured in preruminant lambs after feeding a meal containing either 2.3 or $10.6 \mathrm{~g}$ leucine $/ 100 \mathrm{~g}$ dry matter and were found similar. These two hormones are probably not involved in the effects induced by dietary leucine excess.

L'excès alimentaire de leucine a de nombreux effets chez l'agneau préruminant (ingestion, catabolisme des acides aminés à chaîne ramifiée, renouvellement des protéines) (Papet, 1987). Diverses hormones pourraient être impliquées : le cortisol puisque la sécrétion des glucocorticoïdes est stimulée par la leucine chez le rat (Munro et al., 1963) ; le glucagon qui peut activer la deuxième enzyme de la voie du catabolisme des acides aminés à chaîne ramifiée dans le foie chez le rat (Block et al., 1985). Bien que la leucine soit un acide aminé insulinosécréteur, l'intervention de l'insuline semble faible chez l'agneau préruminant (Papet et al., 1987). Nous avons donc analysé l'influence d'un excès alimentaire de leucine sur la cortisolémie et de la glucagonémie postprandiales chez l'agneau préruminant.

Matériel et méthodes. Deux expériences I (cortisol) et II (glucagon) ont été réalisées sur respectivement 5 et 6 agneaux mâles, de race Limousine, dont la vitesse de croissance était d'environ $280 \mathrm{~g} / \mathrm{j}$. Leurs poids vifs moyens étaient respectivement de 7,0 et $13,5 \mathrm{~kg}$. Ils étaient nourris ad libitum avec un aliment d'allaitement (Agnodor, Union Univor, Paris) à $24 \%$ de protéines $(\mathrm{N} \times 6,25 \mathrm{~g} / 100 \mathrm{~g}$ de matière sèche $(\mathrm{MS}))$ apportées par de la poudre de lait et contenant $2,3 \mathrm{~g}$ de leucine $/ 100 \mathrm{~g}$ MS. Un aliment contenant $10,6 \mathrm{~g}$ de leucine/100 g MS a été obtenu par addition de L-leucine libre (Ajinomoto Co. Inc., Tokyo, Japon) à l'aliment de base. Cet aliment supplémenté a été distribué uniquement pendant $2 \mathrm{j}$ ( $(\mathrm{J}+1$ » et $" \mathrm{~J}+2$ ») au cours de l'expérience 1 et pendant $1 \mathrm{j}(\|\mathrm{J}+1\|)$ lors de l'expérience II. Pendant le jour précédant les excès de leucine ( $J O J)$ ) ainsi que les jours $J+1$ et $J+2$, des prélèvements de sang d'une veine jugulaire ont été réalisés avant et à différents moments après le début du repas du matin (300-500 g de lait). Les animaux sont privés de nourriture entre la fin du repas et la dernière prise de sang. Le glucagon a été dosé par radioimmunologie (kit 10904 Biodata) dans les échantillons de plasma conservés à $-80^{\circ} \mathrm{C}$ en présence d'aprotinine. Le cortisol a été mesuré par radiocompétition et le glucose, par la méthode à la glucose-déshydrogénase (Merck). 
Résultats et discussion. Glycémie. - L'ingestion du repas témoin induit une augmentation postprandiale transitoire de la glycémie d'environ $20 \%$ due à l'absorption du glucose formé à partir du lactose. Cette augmentation n'est pas significativement modifiée lorsque la teneur alimentaire en leucine augmente.

Cortisolémie. - L'ingestion du repas témoin (JO) ainsi que du repas contenant la leucine en excès $(J+1$ et $J+2)$ ne provoque pas de variation significative de la cortisolémie postprandiale (fig. 1). Le pic observé le second jour d'excès de leucine n'est pas significatif. Dans nos conditions expérimentales, la leucine ne semble donc pas modifier la sécrétion du cortisol tandis qu'elle stimule celle des glucocorticoides chez le rat (Munro et al., 1963).

Glucagonémie. - Aucune modification significative de la glucagonémie, qui est faible, n'a été observée après l'ingestion du repas témoin (JO) (fig. 1). Or, chez le veau préruminant un pic a été détecté au cours des 10 min qui suivent l'ingestion d'un repas témoin (Bloom et al., 1975). Aucune variation de la glucagonémie n'a été induite par l'excès alimentaire de leucine chez l'agneau préruminant (fig. 1) bien que d'une part, la leucine ainsi que son premier catabolite stimulent la sécrétion du glucagon sur des pancréas de rats perfusés (Leclercq-Meyer et al., 1985) et d'autre part, les teneurs plasmatiques en ces deux composés soient fortement accrues $(\times 5)$ par l'ingestion du repas supplémenté en leucine (Papet, 1987).
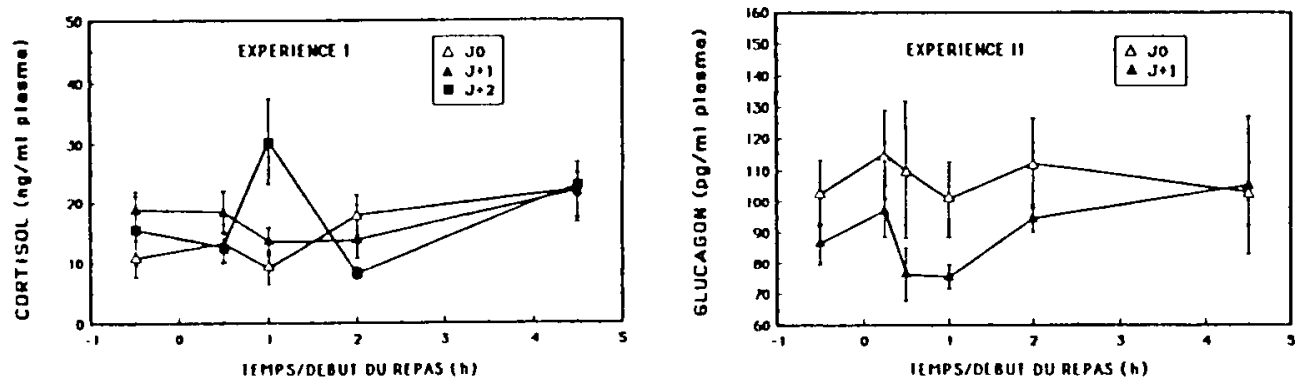

FIG. 1. - Effets de l'augmentation de la teneur alimentaire en leucine de 2,3 (J0) à $10,6 \mathrm{~g} / 100 \mathrm{~g} \mathrm{MS}$ $(\mathrm{J}+1$ et $\mathrm{J}+2)$ sur les cinétiques postprandiales des teneurs plasmatiques en cortisol (expérience 1 ) et en glucagon (expérience II) chez l'agneau préruminant.

L'étude des variations postprandiales des teneurs plasmatiques en glucagon et en cortisol n'a pas permis d'impliquer ces hormones dans les effets nutritionnels et métaboliques induits par un excès alimentaire de leucine chez l'agneau préruminant.

Remerciements. - Nous tenons à remercier M. J. P. Chacornac pour les dosages de glucose.

Block K. P., Heywood B. W., Buse M. G., Harper A. E., $1985 . \quad$ Biochem. J., 232, 593-597.

Bloom S. R., Edwards A. V., Hardy R. N., Malinowska K., Silver M., 1975. J. Physiol., 253, 135-155. Leclercq-Meyer V., Marchand J., Woussen-Colle M. C., Giroix M. H., Malaise W. J., 1985. Endocrinology, 116, 1168-1174.

Munro H. N., Steele M. H., Hutchinson W. C., 1963. Nature, 199, 1182.

Papet I., 1987. Thèse de I'Université Blaise Pascal Clermont II.

Papet I., Breuillé D., Attaix D., Grizard J., Arnal M., 1987. Reprod. Nutr. Dévelop., 27, 297-298. 